\title{
Papel do método Pilates na cognição e autoimagem de idosos: Revisão sistemática
}

\author{
The role of Pilates method in the elderly's cognition and self-image: Systematic review \\ Papel del método Pilates en la cognición y la autoimagen del anciano: Una revisión sistemática
}

Recebido: 23/03/2021 | Revisado: 02/04/2021 | Aceito: 06/04/2021 | Publicado: 17/04/2021

Cinthia Fernanda da Fonseca Silva
ORCID: https://orcid.org/0000-0002-6519-3857
Universidade Federal do Paraná, Brasil
E-mail: fisioterapiacinthia@gmail.com
Ana Caroline de Paula
Onato
ORCID: https://orcid.org/0000-0002-2536-6848
Universidade Federal do Paraná, Brasil
E-mail: anacarolinedp@ gmail.com
Fabiana Maria Alexandre
ORCID: https://orcid.org/0000-0002-2610-4743
Universidade Federal do Paraná, Brasil
E-mail: biamalexx @ gmail.com
Gilson Brun
ORCID: https://orcid.org/0000-0002-9090-0736
Universidade Federal do Paraná, Brasil
E-mail: gilsonbrun1 @gmail.com
Neméia de Oliveira Farias
ORCID: https://orcid.org/0000-0001-5515-8380
Universidade Federal do Paraná, Brasil
E-mail: nemeiafarias@ @otmail.com
Valdomiro de Oliveira
ORCID: https://orcid.org/0000-0002-8709-8471
Universidade Federal do Paraná, Brasil
E-mail: oliveirav457@gmail.com
Gislaine Cristina Vagetti
ORCID: https://orcid.org/0000-0003-0704-1297
Universidade Estadual do Paraná, Brasil
E-mail: gislainevagetti@ @otmail.com

\section{Resumo}

O Pilates é um sistema de exercícios que visa melhora na flexibilidade, resistência física, força, equilíbrio e coordenação motora. Além disso, acredita-se que o método possa contribuir na melhora dos padrões cognitivos e de autoimagem das pessoas idosas, pois o praticante precisa realizar os exercícios de maneira consciente e fluída. O objetivo dessa revisão sistemática foi avaliar o papel do método Pilates na cognição e autoimagem de idosos. Foram realizadas duas revisões sistemáticas nas bases de dados BVS, Pubmed/Medline, Scielo, Web of Science, Cochrane, Eric, Capes e Psycinfo, entre os anos de 2009 a 2019. Como resultado, foram encontrados dois artigos sobre a prática de Pilates na cognição em idosos. Tais estudos demonstraram uma melhora na função cognitiva e função geral de idosos praticantes do método. Sobre a relação entre o Pilates, autoimagem e idosos nenhum artigo foi encontrado. Dessa forma, é aparente a necessidade de mais estudos que contemplem a relação entre a cognição e autoimagem em idosos praticantes de Pilates, já que são fatores que ajudam no desenvolvimento pessoal e qualidade de vida.

Palavras-chave: Idoso; Técnicas de exercício e de movimento; Cognição; Autoimagem.

\begin{abstract}
The Pilates method is an exercise system that pursuit an improvement in flexibility, physical resistance, strength, balance, and motor coordination. Furthermore, it is generally agreed that the method can contribute to the improvement of cognitive and self-image patterns of the elderly, for the practitioner needs to perform the exercises consciously and fluidly. This systematic review aimed to evaluate the role of the Pilates method in the cognition and self-image of the elderly. It was done two systematic reviews in BVS, Pubmed/Medline, Scielo, Web of Science, Cochrane, Eric, Capes, and PsycINFO databases, between 2009 and 2019. As a result, two articles were found on Pilates' practice on cognition in the elderly. These studies evidenced an improvement in the cognitive function and general function of elderly practitioners of the method. About the relationship between Pilates, self-image, and the elderly, no article was found. Thereby, it appears the need for more studies that contemplate the relation of cognition and self-image in the elderly practitioners of the Pilates method, for they are factors that help in personal development and quality of life.
\end{abstract}

Keywords: Elderly; Exercise movement techniques; Cognition; Self-image. 


\begin{abstract}
Resumen
Pilates es un sistema de ejercicios que tiene como objetivo mejorar la flexibilidad, la resistencia física, la fuerza, el equilibrio y la coordinación motora. Además, se cree que el método puede contribuir a mejorar los patrones cognitivos y de autoimagen de las personas mayores, ya que el practicante necesita realizar los ejercicios de manera consciente y fluida. El objetivo de esta revisión sistemática fue evaluar el papel del método Pilates en la cognición y la autoimagen en los ancianos. Se realizaron dos revisiones sistemáticas en las bases de datos BVS, Pubmed / Medline, Scielo, Web of Science, Cochrane, Eric, Capes y Psycinfo, entre los años 2009 a 2019. Como resultado, se encontraron dos artículos sobre la práctica de Pilates en la cognición en los ancianos. Dichos estudios han demostrado una mejora en la función cognitiva y la función general de las personas mayores que practican el método. En cuanto a la relación entre Pilates, autoimagen y personas mayores, no se encontró ningún artículo. Así, se evidencia la necesidad de más estudios que contemplen la relación entre cognición y autoimagen en practicantes de Pilates ancianos, ya que son factores que ayudan en el desarrollo personal y la calidad de vida.

Palabras clave: Anciano; Técnicas de ejercicio y movimiento; Cognición; Auto imagen.
\end{abstract}

\title{
1. Introdução
}

Seguindo uma corrente mundial, no Brasil a população está envelhecendo. Em 2018, o IBGE mostrou que o percentual de pessoas com mais de 65 anos é de 9,2\% (19,2 milhões). Projeções para 2060 aumentam esse percentual para 25,5\% (58,2 milhões de idosos). Pode-se explicar esse fenômeno pela baixa na taxa de fecundidade (1,77 filho por mulher, em 2018) e o aumento da expectativa de vida ao nascer (Instituto Brasileiro de Geografia e Estatística [IBGE], 2018).

Portanto, envelhecer é um processo biológico com consequências psicológicas e que demanda adaptação contínua e compensação para enfrentar as mudanças fisiológicas que ocorrem com o tempo (Freitas et al., 2010). O termo envelhecimento é utilizado para descrever as mudanças morfofuncionais ao longo da vida, ocorridas após a maturação sexual e que progridem comprometendo a capacidade de resposta dos indivíduos ao estresse ambiental e à manutenção da homeostasia. É um processo dinâmico e progressivo ocasionando maior vulnerabilidade e maior incidência de patologias que podem levar à morte (Moreira, 2017).

Essa mudança no perfil demográfico da população trouxe novos desafios aos pesquisadores, já que com isso, houve uma mudança no perfil epidemiológico, ou seja, morbidades que antes eram pouco relatadas passaram a aumentar devido ao envelhecimento. Um exemplo disso é a saúde mental e a cognição, que nesta faixa etária merece atenção diferenciada, pois o declínio cognitivo traz desconforto pessoal devido à perda de autonomia e reflete no aumento dos custos sociais (Camarano \& Kanso, 2017).

Cognição engloba toda a esfera do funcionamento mental, a qual envolve habilidades de pensar, de perceber, de lembrar, de sentir, de raciocinar e de responder aos estímulos externos, ou seja, a maneira pela qual a informação é obtida, codificada e processada (Carneiro, 2015). A importância da integridade dos processos cognitivos e intelectuais, bem como, a continuidade de mecanismos de autorregulação da personalidade é determinante na longevidade e qualidade de vida na velhice. Além da adequação do comportamento e resolução de situações do dia a dia, o aspecto cognitivo também altera a percepção subjetiva do indivíduo, interferindo em sua qualidade de vida (Beckert et al., 2012).

Por percepção subjetiva, pode-se falar sobre a autoimagem. A imagem corporal é a representação e figuração do corpo, formada na mente, ou seja, como a pessoa imagina ser, e desempenha um papel fundamental para os seres humanos. $\mathrm{O}$ esquema corporal consiste na imagem tridimensional que cada um tem de si mesmo, incluindo os aspectos psicológicos, sociológicos e fisiológicos. É o reconhecimento que cada pessoa faz de si, como se sentem, suas potencialidades, sentimentos, atitudes e ideias e surge da interação do indivíduo em suas relações no seu contexto social. A autoimagem dos idosos é um desafio, tanto por ele mesmo quanto pelo modo como a sociedade começa a tratá-lo (Scatolin, 2012).

A imagem corporal começa a se formar desde o nascimento, sendo que o seu desenvolvimento ocorre junto do desenvolvimento sensório motor. A experiência visual tem papel importante na formação da autoimagem, e está intimamente ligada e é vivenciada por meio da ação, do mover-se. As impressões visuais influenciam o esquema corporal e cada um é 
modificado e reestruturado a partir dela (Scatolin, 2012).

Como dito anteriormente, a medida em que se envelhece há a perda progressiva das funcionalidades. Isso altera o processo de informações entre o corpo e cérebro, modificando as sensações de percepção corporal, resultando na interpretação errada dos parâmetros corporais. Uma das formas de preservar a autoimagem do idoso, melhorando sua qualidade de vida e estimulando uma vida plena e realizada perante a sociedade, é a prática de atividades físicas e de lazer. Por meio da atividade física, há a aprendizagem de novos movimentos e isso faz com que o indivíduo utilize a cognição para a realização de diferentes funções executivas (Fonseca, 2012; Gomes et al. Costa, 2017).

Criado por Joseph Hubertus Pilates, ao final da Primeira Guerra Mundial, o Pilates é um sistema de exercícios que visa melhora na flexibilidade, resistência física, força, equilíbrio e coordenação motora (Costa, 2016). Foi primeiramente batizado de Contrologia, pois para Pilates o método resulta do equilíbrio entre o corpo e mente através do controle consciente dos movimentos musculares e corporais pela correta utilização e aplicação dos princípios biomecânicos, aliando o conhecimento dos princípios do equilíbrio e da gravidade durante o repouso e ação corporal (Pilates, 2010). Dessa forma, percebe-se que o método Pilates pertence não somente à atmosfera fitness, mas também à cognição, devido ao uso do controle consciente da coordenação motora.

Pilates (2010) relata que o método não é um sistema aleatório construído somente para produzir músculos salientes, mas que foi concebido e testado por anos, com o intuito de exercitar de maneira correta e cientifica todos os músculos do corpo. Caldwell et al. (2013) relatam que o método Pilates tem o potencial de cultivar a atenção plena (mindfulness), que é uma qualidade mental associada ao bem-estar geral. A atividade física que possui foco na atenção plena contém uma percepção sensorial autorreflexiva, inclui percepção do movimento e orientação espacial, tem foco na respiração e no som da respiração e é a atenção volta-se alinhamento anatômico.

Por ser difundida como uma prática de exercícios sem impactos e mais tranquila, muitos idosos têm procurado o método Pilates em busca de saúde e manutenção das aptidões físicas, porém, na literatura há poucos estudos que indiquem os benefícios do método, especificamente na população idosa (Costa, Schulz, Haas \& Loss, 2016). Compreendendo a necessidade de maior autonomia e de um envelhecimento de qualidade da população idosa, e observando uma lacuna referente a pesquisas relacionando Pilates, idosos, cognição e autoimagem o objetivo dessa revisão sistemática é buscar estudos que tragam a relação dos aspectos cognitivos e de autoimagem com a prática de Pilates por idosos.

\section{Metodologia}

\section{Tipo de estudo}

Para maior aprofundamento na temática dessa pesquisa, optou-se por realizar duas revisões sistemáticas. Em cada revisão foram montadas estratégias de buscas diferentes, com diferentes descritores. A ideia atual de uma revisão de pesquisa realizada de maneira sistemática surgiu na década de 1970, sendo que revisão sistemática se refere ao processo de reunião, avaliação crítica e sintética de resultados de diversos estudos (Costa \& Zoltowski, 2014).

\section{Estratégia de busca}

A identificação dos artigos incluídos nesta revisão foi feita por meio de duas buscas separadas. Essa estratégia foi escolhida para que houvesse uma maior varredura nas bases de dados e para que as variáveis cognição e autoimagem não conflitassem entre si, pois a busca seria restringida pela excessiva delimitação de tema caso ambas fossem colocadas na mesma string.

A busca de artigos foi realizada em oito bases de dados oficiais, tanto da área da Educação quanto da Saúde, sendo elas: Biblioteca Virtual de Saúde (BVS), Pubmed/Medline, Scientific Eletronic Library Online (Scielo), Web of Science, 
Education Resources Information Center (Eric), Cochrane Library, Periódico Capes e Psycinfo. Foram utilizados somente artigos, sendo excluídas teses, dissertações e monografias.

O corte temporal foi delimitado em dez anos (janeiro de 2009 a abril de 2019), considerando artigos publicados nos idiomas português, inglês e espanhol, visto que textos e periódicos de maior impacto acadêmico são publicados ou traduzidos nesses idiomas. Para a investigação, foram utilizados os descritores encontrados nos Descritores em Ciências da Saúde (DeCS), Medical Subject Headings (MeSH) e Thesaurus. Após a busca e escolha dos artigos, foi utilizada a ferramenta de análise de qualidade de artigos, o checklist de Downs \& Black (Downs \& Black, 1998). Essa ferramenta foi elencada porque somente artigos de intervenção foram encontrados.

Como auxílio nas pesquisas, foi utilizado o programa State of the Art through Systematic Review (START), desenvolvido pelo Laboratório de Pesquisa em Engenharia de Software (Lapes) da Universidade Federal de São Carlos, que tem por objetivo dar suporte ao pesquisador na revisão sistemática. As buscas foram realizadas utilizando descritores nas línguas portuguesa, inglesa e espanhola. Na busca sistematiza I (BS I), foram utilizados os seguintes descritores: Cognição (Cognition, Cognición, Memória, Linguagem, Percepção visioespacial, Atenção e Funções executivas); Idoso (Anciano, Aged, Older Adults) e Técnicas de exercício e de movimento (Exercise movement techniques, Técnicas de ejercício con movimentos, Método Pilates, Pilates). Na busca sistematizada II (BS II), foram utilizados os seguintes descritores: Autoimagem (Selfconcept, Self, Self-psychology); Idoso (Anciano, Aged, Older Adults) e Técnicas de exercício e de movimento (Exercise movement techniques, Técnicas de ejercício con movimentos, Método Pilates, Pilates). Foram utilizados os operadores booleanos "AND" e "OR" para a combinação dos descritores. Esta revisão foi realizada em pares (peer-review).

As etapas de avaliação dos artigos encontrados foram separadas em três partes: (1) exclusão dos estudos que não contemplaram os critérios de inclusão e exclusão na leitura do título dos artigos; (2) exclusão dos estudos que não contemplaram os critérios de inclusão e exclusão na leitura do resumo dos artigos. A partir desse momento a seleção ocorreu por meio de duas pessoas (peer-review). (3) leitura integral dos artigos selecionados, para avaliar se ainda contemplam os critérios de inclusão e exclusão. Após isso, foi feita uma qualificação dos estudos utilizando o checklist de Downs \& Black.

Todas as etapas desse artigo de revisão sistemática obedeceram ao checklist PRISMA (Preferred Reporting Items for Systematic Reviews and Meta-Analyses), tais como critérios de inclusão e exclusão, informações das bases de dados, estratégias de busca, uso de ferramenta para auxílio na seleção dos artigos, revisão por pares e especificação dos métodos utilizados nas buscas.

\section{Critérios de inclusão}

Foram considerados os seguintes critérios de inclusão: (a) artigos originais publicados em periódicos peer-reviewed; (b) estudos publicados a partir de janeiro de 2009; (c) participantes do estudo com idade superior ou igual a 60 anos.

\section{Critérios de exclusão}

Foram considerados os seguintes critérios de exclusão: (a) teses, dissertações e monografias; (b) estudos publicados antes de janeiro de 2009; (c) estudos que não atingiram a pontuação mínima de 18 pontos no checklist de Downs \& Black; (d) estudos em duplicidade encontrados em diferentes bases de dados; (e) estudos com participantes que apresentem transtornos cognitivos (exceto os leves); (f) estudos fora da temática dessa pesquisa.

\section{Extração dos dados}

Os estudos incluídos nesta revisão serão dispostos em um quadro com as seguintes informações: autor, ano, pontuação de Downs \& Black, país, periódico e objetivo. Após o Quadro 1, os artigos serão relatados mais detalhadamente. 


\section{Resultados}

\section{Busca Sistematizada I - Pilates, cognição e idosos}

A Figura 1 mostra o fluxograma descrevendo o processo de busca e seleção dos artigos referentes a BS I. Foram encontrados 142 artigos no total. Após a leitura dos títulos, foram elencados 13 artigos, foi realizada a leitura dos resumos e restaram 3 artigos. Desses três, foram aceitos dois após a leitura na íntegra.

Figura 1 - busca e seleção dos artigos da BS I

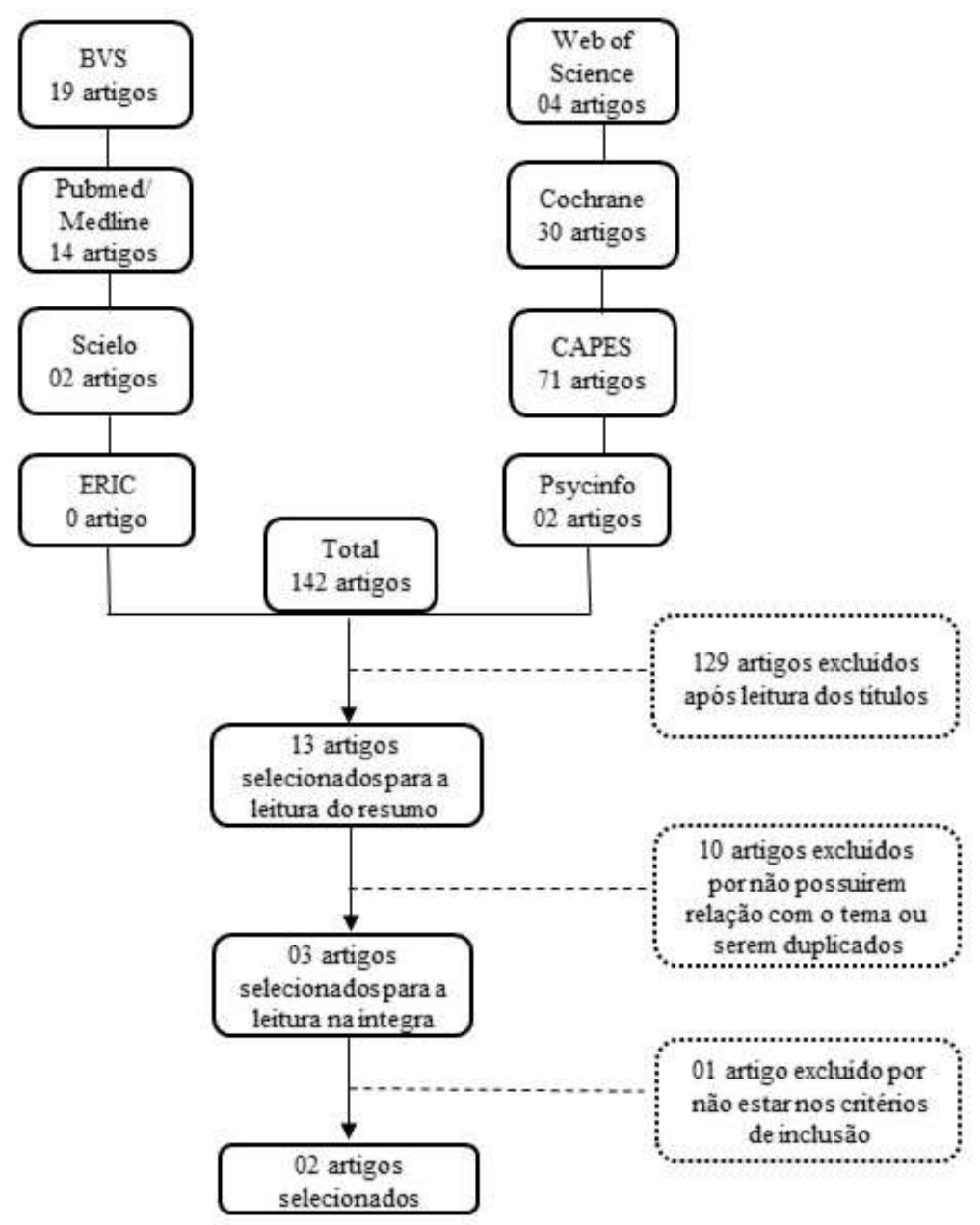

Fonte: Autores (2019).

Abaixo, segue o quadro relacionando o autor, ano, pontuação de Downs \& Black, país, periódico e o objetivo dos estudos. 
Quadro 1 - dados dos artigos da BS I

\begin{tabular}{|l|l|l|}
\hline \multicolumn{1}{|c|}{ Autor/Ano/DB* } & \multicolumn{1}{|c|}{ País/Periódico } & \multicolumn{1}{c|}{ Objetivo } \\
\hline $\begin{array}{l}\text { JURAKIC et al./ } \\
\text { 2017/ DB 18 }\end{array}$ & $\begin{array}{l}\text { Suíça/Aging Clinical and } \\
\text { Experimental Research }\end{array}$ & $\begin{array}{l}\text { Fornecer provas preliminares sobre os } \\
\text { efeitos de dois tipos de treinamento não } \\
\text { aeróbico em funções cognitivas em } \\
\text { mulheres idosas que sofrem de } \\
\text { comprometimento cognitivo leve. }\end{array}$ \\
\hline $\begin{array}{l}\text { CARRASCO- } \\
\text { POYATOS et } \\
\text { al./2019/ DB 22 }\end{array}$ & Holanda/Physiology and Behaviour & $\begin{array}{l}\text { Analisar dois programas de treinamento na } \\
\text { funcionalidade, equilíbrio e composição } \\
\text { corporal em idosas e determinar a } \\
\text { influência da função cognitiva. }\end{array}$ \\
\hline
\end{tabular}

*DB= Downs \& Black. Fonte: Autores (2019).

No estudo de Jurakic et al. (2017), o objetivo foi prover evidências dos efeitos de exercícios não aeróbicos nas funções cognitivas de 28 mulheres idosas (idades de 66 a 78 anos), com comprometimento cognitivo leve. Para isso, elas foram separadas aleatoriamente em dois grupos, um deles realizou exercícios em um aparelho denominado HUBER e o outro grupo foi o de Pilates. Os treinos ocorreram três vezes por semana durante 8 semanas. O instrumento utilizado para avaliar alguns domínios cognitivos foi o Montreal Cognitive Assessment (MoCA), sendo realizado no pré e no pós-intervenção. Os resultados mostraram que tanto os exercícios que combinam treino de equilíbrio e resistência do core quanto os de Pilates melhoraram as funções cognitivas globais, bem como, algumas específicas. Especificamente na prática do Pilates, foi observado uma melhora nos domínios de evocação tardia (memória de curto prazo). A linguagem e a abstração tiveram efeito positivo nos dois grupos. Dessa maneira, atividades físicas não aeróbicas podem trazer benefícios para idosos com leve comprometimento cognitivo.

Já o estudo de Carrasco-Poyatos et al. (2019) teve por objetivo analisar o efeito de dois programas de exercício físico na autonomia funcional, equilíbrio e composição corporal de idosas, bem como, determinar a influência das funções cognitivas nesses aspectos. Para isso, 60 mulheres idosas (60 a 80 anos) foram divididas em três grupos, aleatoriamente, sendo um grupo de prática de Pilates, um grupo de prática de musculação e outro que não praticava exercício físico. Cada grupo ficou com 20 participantes. Os programas de treinamento foram implementados por 18 semanas. Os testes realizados foram: protocolo GDLAM para avaliar a autonomia funcional, Mini Exame do Estado Mental (MEEM) para avaliar as funções cognitivas, a composição corporal foi avaliada por meio de uma densitometria óssea por raio-X de dupla energia e o equilíbrio estático foi testado através do teste de duas pernas e foi avaliado usando uma plataforma de força portátil.

O achado principal da intervenção foi que as 18 semanas foram suficientes para melhorar significativamente a autonomia funcional das idosas, além do que, as participantes do grupo Pilates adquiriram um melhor resultado na condição de função geral do que as do grupo de musculação. Em relação à cognição, foi encontrado que a melhora na função cognitiva leva a um aumento na agilidade e equilíbrio dinâmico, bem como, a uma melhor condição na função geral (Carrasco-Poyatos et al., 2019).

Devido à escassez de estudos encontrados - somente dois artigos - optou-se por realizar uma busca manual nas referências bibliográficas dessas pesquisas a fim de verificar se algum trabalho passou despercebido, respeitando o corte temporal de dez anos.

Entretanto, não havia nenhum outro estudo relacionando Pilates, idosos e cognição na lista de referências. Porém, como o método Pilates é uma atividade física, decidiu-se elencar artigos que trouxeram as variáveis idosos, cognição e exercícios físicos para melhor embasamento dos autores no momento da discussão. Dessa forma, segue o Quadro 2 relacionando o autor, ano, país, periódico, tipo e o objetivo do estudo dos artigos encontrados nas referências bibliográficas. 
Quadro 2 - Artigos encontrados nas referências

\begin{tabular}{|c|c|c|c|}
\hline Autor/Ano & País/Periódico & Tipo de estudo & Objetivo \\
\hline $\begin{array}{l}\text { SOFI, F. et al./ } \\
2010\end{array}$ & $\begin{array}{l}\text { Suécia/Journal of } \\
\text { Internal Medicine }\end{array}$ & Metanálise & $\begin{array}{l}\text { Investigar a associação entre } \\
\text { atividade física e o risco de declínio } \\
\text { cognitivo em participantes sem } \\
\text { demência. }\end{array}$ \\
\hline $\begin{array}{l}\text { CHANG, Y. et al./ } \\
2012\end{array}$ & $\begin{array}{l}\text { Inglaterra/Aging } \\
\text { and Physical } \\
\text { Activity }\end{array}$ & $\begin{array}{l}\text { Revisão de } \\
\text { literatura }\end{array}$ & $\begin{array}{l}\text { Examinar o papel de exercícios de } \\
\text { treinamento de resistência na } \\
\text { cognição de idosos saudáveis. }\end{array}$ \\
\hline $\begin{array}{l}\text { NAGAMATSU, } \\
\text { L.S. et al./ } 2012\end{array}$ & $\begin{array}{l}\text { Canadál Archives } \\
\text { of Internal } \\
\text { Medicine }\end{array}$ & $\begin{array}{l}\text { Estudo } \\
\text { randomizado } \\
\text { controlado }\end{array}$ & $\begin{array}{l}\text { Realizar um ensaio clínico } \\
\text { randomizado } \\
\text { para fornecer evidências de eficácia } \\
\text { tanto de exercícios de resistência } \\
\text { quanto do treinamento aeróbico na } \\
\text { melhora das funções cognitivas } \\
\text { executivas. Examinar os efeitos dos } \\
\text { dois tipos de exercício na memória } \\
\text { associativa, habilidade de resolução } \\
\text { de problemas diários, padrões } \\
\text { regionais de plasticidade cerebral } \\
\text { funcional e na função física. }\end{array}$ \\
\hline $\begin{array}{l}\text { GATES, N. et al./ } \\
2013\end{array}$ & $\begin{array}{l}\text { Estados Unidos/ } \\
\text { American Journal } \\
\text { of Geriatric } \\
\text { Psychiatry }\end{array}$ & Metanálise & $\begin{array}{l}\text { Avaliar a eficácia do exercício a } \\
\text { cognição de idosos com } \\
\text { comprometimento cognitivo leve. }\end{array}$ \\
\hline $\begin{array}{l}\text { ÖHMAN, H. et al./ } \\
2014\end{array}$ & $\begin{array}{l}\text { Suíça/ Dementia } \\
\text { and Geriatric } \\
\text { Cognitive } \\
\text { Disorders }\end{array}$ & Revisão sistemática & $\begin{array}{l}\text { Realizar uma revisão sistemática, } \\
\text { por meio de estudos randomizados } \\
\text { controlados, dos efeitos de } \\
\text { exercícios físicos na cognição de } \\
\text { idosos com comprometimento } \\
\text { cognitivo leve ou demência. }\end{array}$ \\
\hline $\begin{array}{l}\text { SÁEZ DE } \\
\text { ASTEASU, M.L. } \\
\text { et al./ } 2017\end{array}$ & $\begin{array}{l}\text { Holanda/ Ageing } \\
\text { Research Reviews }\end{array}$ & Revisão sistemática & $\begin{array}{l}\text { Analisar os efeitos de diferentes } \\
\text { modalidades de exercícios físicos } \\
\text { como treino aeróbico, de resistência } \\
\text { e de multicomponentes na cognição } \\
\text { em idosos saudáveis sem } \\
\text { comprometimento cognitivo. } \\
\text { Esclarecer as discrepâncias } \\
\text { observadas entre estudos com } \\
\text { animais, epidemiológicos e } \\
\text { transversais em relação aos } \\
\text { resultados menos consistentes } \\
\text { observados nos estudos } \\
\text { randomizados controlados. }\end{array}$ \\
\hline
\end{tabular}

Fonte: Autores (2019).

Observa-se que os artigos são da Europa e da América do Norte. Assim como os estudo encontrados na BS I, não houve nenhum artigo brasileiro ou sul-americano. Dessa forma, percebe-se que há um campo fértil para a pesquisa quando se pensa no papel do método Pilates na cognição de idosos. Os dados encontrados nas buscas serão discutidos e relacionados na Discussão. 


\section{Busca sistematizada II - Pilates, autoimagem e idosos.}

A Figura 2 mostra o fluxograma com o processo de busca e seleção de artigos referentes à BS II. Foram encontrados 708 artigos no total. Após a leitura dos títulos, foram elencados 14 artigos para a leitura dos resumos, depois dessa análise, restou um artigo. Após a leitura total, o artigo foi descartado por não tratar da autoimagem.

Figura 2 - Busca e seleção dos artigos da BS II.

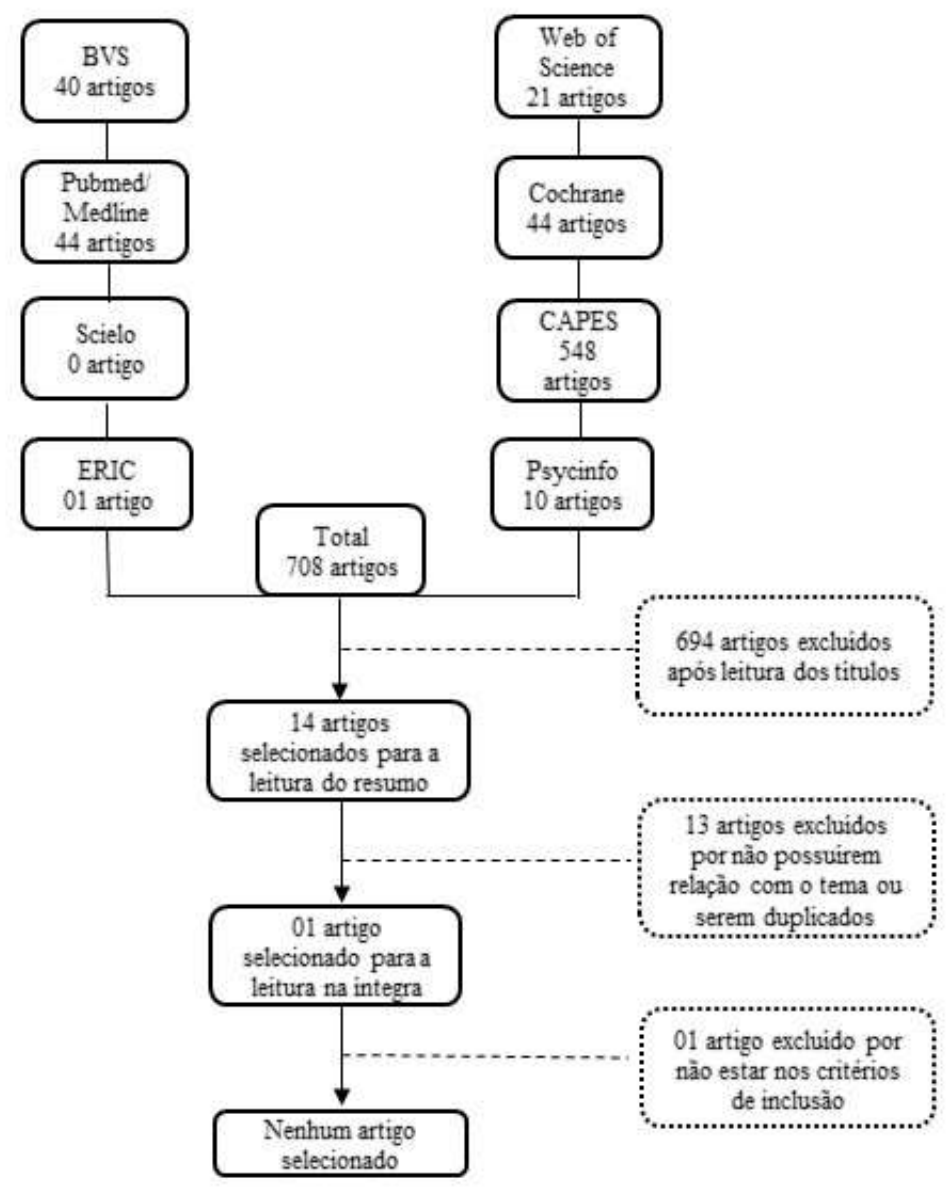

Fonte: Autores (2019).

O artigo selecionado para a leitura na íntegra, de autoria de Puleo (2017), fala sobre como o Pilates e as intervenções que buscam ensinar movimentos conscientes podem auxiliar em um envelhecimento de qualidade. Porém, não traz nada sobre a autoimagem, mas somente sobre a autoeficácia e a atividade física, que não estão no escopo dessa pesquisa. Talvez isso ocorra pelos poucos instrumentos de avaliação de autoimagem, especificamente em idosos, o que pode dificultar uma pesquisa nesse campo.

\section{Discussão}

Após a realização da revisão sistemática, constatou-se que há poucos estudos relacionando o Pilates com a cognição e autoimagem em idosos. Assim, para a construção da discussão sobre Pilates e cognição, foram utilizados os artigos encontrados tanto na BS I quanto na busca manual das referências. Já na discussão sobre Pilates e autoimagem, devido à falta 
de estudos resultantes da BS II, foram abordados estudos sobre autoimagem e outros exercícios físicos em idosos, bem como, estudos que explorem a importância da imagem corporal.

\section{Pilates, cognição e idosos}

Os dois artigos encontrados na BS I foram publicados em revistas europeias, as quais possuem enfoque na área da saúde. Ao compreender a importância da educação na aprendizagem de movimentos corporais, e em como isso pode beneficiar o indivíduo para uma melhora nas habilidades cognitivas, assume-se a necessidade de que mais pesquisas envolvendo atividade física e saúde sejam realizadas (Gomes et al., 2017; Benedetti et al., 2003; Fonseca, 2012).

Em geral, os resultados relativos à cognição e prática de atividade física foram semelhantes nos grupos de praticantes de Pilates e de praticantes de outras modalidades de exercício. Como exemplo, tem-se o estudo de Carrasco-Poyatos et al. (2019), na qual explicou-se que uma melhor função cognitiva pode levar a um aumento na agilidade e no equilíbrio dinâmico, consequentemente, melhorando a função geral em idosos.

Em relação à memória, um grupo de praticantes de Pilates obteve melhora nos domínios de evocação tardia e memória de curto prazo. Já nos domínios linguagem e abstração, o efeito positivo foi observado tanto no grupo Pilates, quanto no de prática de atividade física diversa (Jurakic et al., 2017). Ainda sobre a memória, na pesquisa realizada por Nagamatsu et al. (2012) em mulheres idosas (65 a 75 anos), obteve-se resposta de melhora da atenção seletiva/resolução de conflitos, nos padrões de memória associativa e regional e de plasticidade cerebral funcional após seis meses de treinamento de resistência.

Estudos sobre os efeitos do treinamento de resistência na cognição em idosos ainda são recentes (Chang et al., 2012). Talvez isso ocorra pelo fato de a velhice ser vista, ainda, de uma maneira frágil, devido às mudanças morfofuncionais que levam o indivíduo a apresentar maior vulnerabilidade física (Moreira, 2017). Entretanto, embora haja resultados conflitantes sobre o papel desse treinamento na prevenção do declínio cognitivo com a idade, muitos estudos demonstraram efeitos benéficos dessa prática em funções cognitivas específicas (Chang et al., 2012).

Foram encontrados alguns estudos com modelo de metanálise nessa revisão. Alguns pesquisaram sobre os efeitos ou a associação das atividades físicas na cognição, ou no declínio cognitivo em idosos com comprometimento cognitivo leve (CCL) ou com demência, outros, em idosos sadios. Alguns autores defendem que são escassas as pesquisas em idosos com CCL, sendo a maioria com idosos saudáveis ou bastante comprometidos (Gates et al.,2013).

Diversos foram as modalidades de prática de atividade física presentes nas metanálises, tais como: exercícios aeróbicos, de resistência, exercícios combinados de resistência, equilíbrio e aeróbicos, de equilíbrio e coordenação e de multicomponentes (Gates et al., 2013; Saez de Asteasu et al., 2017). Os artigos que compuseram a metanálise foram de ensaios clínicos randomizados (ECR).

Em idosos com CCL, ou demência, houve um achado de uma melhora no treinamento de resistência, após seis meses, em idosas de 70 a 80 anos com provável CCL (Nagamatsu et al., 2012). Além disso, outros autores concluíram que houve alguns efeitos positivos na cognição de idosos com CCL praticantes de exercício, tais como, na função cognitiva global, funções executivas, atenção e recuperação tardia (Öhman et al., 2014).

Ao contrário dos achados acima, em Gates et al. (2013) a vasta maioria dos resultados (92\%) não foram significativos, ou seja, não mostraram evidências fortes ou consistentes de que alguma das modalidades de exercícios auxiliou na melhora cognitiva em idosos com CCL ou risco de demência, bem como, nos idosos com demência não foi encontrado nenhum efeito significativo (Öhman et al., 2014).

Apesar de ter sido relatado que alguns pesquisadores sentem falta de mais estudos em idosos com CCL ou demência, para Sofi et al., (2011), são poucos os estudos que investigam a relação entre o estilo de vida ativo e o desempenho cognitivo em participantes saudáveis, já que se fala muito nos transtornos cognitivos e pouco no envelhecimento saudável. 
Em idosos sadios, treze dos vinte e um estudos inclusos na revisão reportaram algumas melhoras cognitivas significativas no grupo de exercício em pelo menos um domínio (memória ou função executiva) (Saez de Asteasu et al., 2017). Apesar disso, não foram encontradas diferenças significativas entre grupos para melhores resultados cognitivos, assim como em outros estudos (Gates et al., 2013; Öhman et al., 2014). Isso pode ser devido à grande variedade de exercícios e de protocolos utilizados nos ECR, o que dificulta uma análise estatística para melhor compreensão da relação entre exercício físico e performance cognitiva.

Indo de encontro a resultados anteriores, numa metanálise com estudos de coorte prospectivos o resultado foi que indivíduos com alto nível de atividade física tiveram risco reduzido de 38\% de declínio cognitivo em relação aos considerados sedentários. Quando considerados os níveis baixos e moderados de atividade física, foram observados fatores de proteção contra o declínio cognitivo (Sofi et al., 2011).

Observa-se que não há um consenso na ciência no assunto da relação da atividade física e cognição, apesar de haver estudos com resultados positivos nessa relação, há alguns que não conseguiram mostrar um achado específico.

\section{Pilates, autoimagem e idosos}

Com o envelhecimento ocorrem diversas mudanças na vida do ser humano, desde as alterações físicas e psicológicas, quanto as sociais e culturais. Entre essas alterações, está a da imagem corporal. Ter uma autoimagem agradável pode influenciar no comportamento da pessoa, melhorando seu desempenho em termos físicos e sociais. Entretanto, as incapacidades físicas resultantes do envelhecimento podem afetar a imagem corporal, já que o corpo é um suporte da identidade individual e social, auxiliando nas relações sociais (Rocha, 2014).

O movimento é o canal pelo qual a pessoa aprende sobre o próprio corpo, pois elabora-se a imagem corporal de acordo com as experiências adquiridas através das ações, ou, do movimento. Dessa forma, a prática de exercício físico é importante pois são meios de diminuir a forma rígida do modelo postural do corpo, levando a uma alteração psíquica, pois o indivíduo tem a capacidade de mudar a autoimagem continuamente (Scatolin, 2012). Além do mais, a obesidade e humor depressivo mostraram-se significativamente associadas à insatisfação corporal (Rocha, 2014).

A construção da imagem corporal se dá também por meio da vivência entre duas ou mais pessoas, pois dessa forma, há a incorporação de diversas partes das imagens dos corpos dos outros e vice-versa, tornando essa imagem um fenômeno social (Barros, 2005). Como exemplo disso, há um achado de pesquisa de Benedetti et al., (2003) que, após uma intervenção com exercícios físicos sistemáticos em idosos de uma Instituição de Longa Permanência (ILP), que os participantes do grupo controle apresentaram diferenças na percepção da autoimagem. Isso pode acontecer pelo fato de que os idosos do grupo controle, mesmo não participando ativamente das atividades, acompanhavam a rotina da intervenção e assistiam às aulas, vivenciando o movimento corporal de seus companheiros (Barros, 2005).

Em um estudo de Moura e Souza (2012) sobre autoimagem, socialização e lazer, é colocado que a autoimagem e a socialização são constructos interligados na esfera humana, pois como visto anteriormente, a imagem que a pessoa faz de si é influenciada pela forma que os outros a veem, bem como, influencia a maneira como os outros irão enxergá-la (Barros, 2005; Benedetti et al., 2003).

Mazo et al. (2006) realizaram um teste para verificar a autoestima e autoimagem de idosos praticantes de hidroginástica e a relação dessas variáveis com os fatores motivacionais de ingresso e permanência dos idosos no programa. O questionário utilizado foi o proposto por Steglich (1978). Como resultado houve que a maioria dos idosos participantes do programa de hidroginástica apresentaram elevada autoestima e autoimagem e, ao relacionar o motivo de ingresso e permanência no programa com as variáveis, observou-se uma relação significativa entre o motivo de permanência e a autoimagem. Supõe-se que uma elevada autoimagem esteja associada à percepção que os idosos tem dos benefícios da 
hidroginástica para a saúde física e mental, bem como, na socialização e sensação de bem-estar. Este resultado demonstra, mais uma vez, a importância que manter uma vida social tem para a autoimagem do idoso (Barros, 2005; Benedetti et al., 2003; Moura \& Souza, 2012).

Quando se fala em satisfação com o corpo, chegou-se a resultados no qual 66\% das participantes do estudo composto por mulheres idosas - estão, de forma geral, insatisfeitas com seu corpo (Fonseca et al., 2014). A imagem corporal pode sofrer distorções durante o envelhecimento, que podem ser reforçadas por estereótipos negativos sobre a velhice, tais como incapacidade, inutilidade e incompetência. Esses fatores podem levar o idoso a se sentir deprimido e rejeitado, piorando sua qualidade de vida, além de que, já foram encontradas associações entre a autoimagem e a saúde mental nessa população (Rocha \& Terra, 2013).

Devido a isso, a elevada autoestima, percebida entre as idosas que realizam alguma atividade física, sugere que a prática de atividades corporais é um elemento que tem influência positiva na vida da pessoa idosa, uma vez que contribui no desenvolvimento emocional e, consequentemente, na autoestima e autoimagem (Fonseca et al., 2014).

\section{Limitações}

Este estudo apresentou limitações como: o corte temporal para a busca, de dez anos (2009 a 2019), pode ter deixado alguns artigos de fora; a exclusão de dados não publicados, de teses e dissertações que podem tratar desses temas e a possível inclusão de alguma outra base de dados científicos ou outros descritores no processo de pesquisa, mesmo as bases e descritores escolhidos nesse estudo sendo de ampla divulgação e concentração de pesquisas acadêmicas.

\section{Considerações Finais}

Essa revisão sistemática referente a pesquisas relacionando Pilates, idosos, cognição e autoimagem incluiu dois artigos, referente à cognição e Pilates e, nenhum artigo foi encontrado com relação ao Pilates e autoimagem em idosos. Devido a isso, foram incorporados artigos encontrados manualmente nas referências bibliográficas da BS I e outros estudos sobre autoimagem para a construção da discussão.

Nas pesquisas onde foi utilizado o método Pilates como exercício físico, houve uma melhora na função cognitiva, bem como, na função geral do idoso. No restante dos estudos envolvendo diversos tipos de exercício físico e a cognição também foi relatada alguma melhora em alguns domínios da função cognitiva, porém a maioria dos resultados foi de uma relação não significativa. Alguns autores colocam que esse resultado pode ser pelo fato de os estudos apresentarem erros metodológicos ou por apresentarem diversos tipos de protocolos de intervenção, dificultando uma análise estatística viável em uma metanálises (Gates et al., 2013; Saez de Asteasu, et al., 2017).

O resultado com poucos estudos na temática desse artigo pode ser devido ao fato de que aspectos psicológicos e educacionais são menos abordados em pesquisas relacionadas com Pilates e idosos. Apesar de haverem pesquisas com diversos tipos de exercícios físicos, e sendo o Pilates um método que agrupa exercícios de força, flexibilidade e coordenação motora, nenhum deles atua com o intuito do método, que é o de aliar o controle consciente de todos os movimentos corporais, para que ocorra uma atividade física com plena consciência corporal, unido mente e corpo.

A partir do evidenciado, percebe-se a necessidade de mais estudos que contemplem a relação do método Pilates com a função cognitiva e aspectos da autoimagem na população idosa, pois são fatores que auxiliam no desenvolvimento pessoal e na manutenção e melhoria da qualidade de vida. Sugere-se que estudos futuros sejam realizados com maior corte temporal e em mais bases de dados, e sem restrição de modalidade de pesquisa para novos achados. 


\section{Referências}

Barros, D. (2005). Imagem corporal: a descoberta de si mesmo. História, Ciências, Saúde, 12(2), 547-554. https://doi.org/10.1590/S0104-59702005000200020

Beckert, M., Irigaray, T.Q. \& Trentini, C. M. (2012). Qualidade de vida, cognição e desempenho nas funções executivas de idosos. Estudos de Psicologia, 29(2), 155-162. https://doi.org/10.1590/S0103-166X2012000200001 .

Benedetti, T. B. \& Petroski, E. L. (2003). Exercícios físicos, auto-imagem e auto-estima em idosos asilados. Revista Brasileira de Cineantropometria \& Desenvolvimento Humano, 5(2), 69-74. https://pesquisa.bvsalud.org/portal/resource/pt/lil-476443?lang=es

Caldwell, K., Adams,M., Quin, R., Harrison, M. \& Greeson, J. (2013). Pilates, Mindfulness and Somatic Education. J Dance Somat Pract, 5(2), 141-153. https://www.ncbi.nlm.nih.gov/pmc/articles/PMC4198945/

Camarano, A. A. \& Kanso, S. (2017). Envelhecimento da população brasileira: uma contribuição demográfica. In: Freitas. In: Freitas, E. V., Py, L., Neri, A. L., Cançado, F. A. X. C., Gorzoni, M. L. \& Doll, J. (Org). Tratado de Geriatria e Gerontologia (4a ed.), pp. 83-103. Guanabara Koogan.

Carneiro, M. (2015). Efeito do treino com exergames no desempenho cognitivo e funcional de idosos sem demência. [Dissertação de mestrado. Universidade Federal da Paraíba].

Carrasco-Poyatos, M., Rubio-Arias, J., Ballesta-Garcia, I., \& Ramos-Campos, D. (2019). Pilates vs. muscular training in older woman-effects in functional factors and the cognitive interaction: a randomized controlled trial. Physiol. Beh., 201, pp.157-164. https://www.ncbi.nlm.nih.gov/pubmed/30529737

Chang, Y., Pan, C., Chen, F., Tsai, C. \& Huang, C. (2012). Effect of resistance-exercise training on cognitive function in healthy older adults: a review. Journal of Aging ans Physical Activity, 20, pp.497-517. 10.1123/japa.20.4.497

Costa, A. B. \& Zoltowski, A. P. C. (2014). Como escrever um artigo de revisão sistemática. In: Koller, S. H, Couto, M. C. P. P \& Von Hohendorf, J. Manual de produção científica (pp. 55-70). Penso.

Costa, L. M. R., Schulz, A., Haas, A. N. \& Loss, J. (2016). Os efeitos do método Pilates aplicado à população idosa: uma revisão integrativa. Revista Brasileira de Geriatria e Gerontologia, 19(4), pp. 695-702. https://doi.org/10.1590/1809-98232016019.150142

Costa, T. (2016). Comparação da aptidão funcional e percepção de qualidade de vida em idosas praticantes e não praticantes do método Pilates. [Dissertação de mestrado. Universidade Federal do Paranál.

Downs, S. H. \& Black, N. (1998). The feasibility of creating a checklist for the assessment of the methodological quality both of randomised and nonrandomised studies of health care interventions. Journal of Epidemiology and Community Health, 52, pp. 377-384. 10.1123/japa.2016-0094

Fonseca, C. C., Gama, E. F., Thurm, B.E., Pereira, E. S., Limongeli, A. M. A. \& Miranda, M. L. J. (2012). Benefícios da estimulação perceptual corporal no esquema corporal de idosos. Revista Brasileira de Geriatria e Gerontologia, 15(2), 353-364. https://doi.org/10.1590/S1809-98232012000200017 .

Fonseca, C. C., Chaves, E. C. L., Pereira, S. S., Barp, M., Moreira, A. M. \& Nogueira, D. A. (2014). Autoestima e satisfação corporal em idosas praticantes e não praticantes de atividades corporais. Revista de Educação Física/UEM, 25(3), 429-439. https://doi.org/10.4025/reveducfis.v25i3.22050 .

Freitas, M. C., Queiroz, T. A. \& Sousa, J. A. V. (2010). O significado da velhice e a experiência de envelhecer para os idosos. Revista de Escola de Enfermagem da USP, 44(2), 407-412. http://dx.doi.org/10.1590/S0080-62342010000200024.

Gates N., Singh, M. A. F., Sachddev, P. S. \& Valenzuela, M. (2013). The effect of exercise training on cognitive function in older adults with mild cognitive impairment: a meta-analysis of randomizes controlled trials. American Journal os Geriatric Psychiatry, 21(11), 10886-1097. 10.1016/j.jagp.2013.02.018

Gomes, F. R. H., Vagetti, G. C., \& Oliveira, V. (2017). Envelhecimento Humano: cognição, qualidadede vida e atividade física. Appris.

Instituto Brasileiro de Geografia e Estatística. (2018). Brasil em números. https://biblioteca.ibge.gov.br/biblioteca-catalogo?id=72\&view=detalhes

Jurakic, Z. G., Krizanic, V., Sarabon, N. \& Markovic, G. (2017). Effects of feedback-based balance and core resistance training vs. Pilates training on cognitive functions in older women with mild cognitive impairment: a pilot randomized controlled trial. Aging Clinical and Experimental Research, 29(6), pp. 1295-1298. https://www.ncbi.nlm.nih.gov/pubmed/28251569

Mazo, G. Z.; Cardoso, F. L. \& Aguiar, D. L (2006). Programa de hidroginástica para idosos: motivação, auto-estima e auto-imagem. Revista Brasileira de Cineantropometria e Desempenho Humano 8(2), 67-72. https://periodicos.ufsc.br/index.php/rbcdh/article/view/3883/16671.

Moreira, V.G. (2017). Biologia do envelhecimento. In: Freitas, E. V., Py, L., Neri, A. L., Cançado, F. A. X. C., Gorzoni, M. L. \& Doll, J. (Org). Tratado de Geriatria e Gerontologia (4a ed.), pp. 13-22. Guanabara Koogan.

Moura, G. A. \& Souza, L. K. (2012). Autoimagem, socialização, tempo livre e lazer: quatro desafios à velhice. Textos \& Contextos, 11(1), pp. 172-183. https://revistaseletronicas.pucrs.br/ojs/index.php/fass/article/view/9492

Nagamatsu L. S, Handy, T. C., Hsu, C. L., Voss, M. \& Liu-Ambroise, T. (2012). Resistance training promotes cognitive and functional brain plasticity in seniors with probable mild cognitive impairment: a 6-month randomized controlled trial. Arch. Intern. Med, 172(8), 666-668. https://www.ncbi.nlm.nih.gov/pmc/articles/PMC3514552/.

Öhman H., Savikko, N., Strandberg, T. E. \& Pitkälä, K. H. (2014). Effect of physical exercise on cognitive performance in older adults with mild cognitive impairment or dementia: a systematic review. Dement Geriatr Cogn Disord, 38(5-6), 347-365. https://www.ncbi.nlm.nih.gov/pubmed/25171577

Pilates, J. H. (2010). A obra completa de Joseph Pilates: sua saúde e o retorno à vida pela contrologia. Phorte. 
Research, Society and Development, v. 10, n. 4, e45010414209, 2021

(CC BY 4.0) | ISSN 2525-3409 | DOI: http://dx.doi.org/10.33448/rsd-v10i4.14209

Puleo, J. (2017). Aging and the cost of inactivity. How Pilates and mindful movement interventions may help stem the tide. Topics in Geriatric Rehabilitation, 33(1), pp. 2-8. https://www.nursingcenter.com/journalarticle?Article_ID=3971158\&Journal_ID=515682\&Issue_ID=3971153

Rocha, L. M. B. C. R. M. (2014). Autopercepção do envelhecimento, autoimagem corporal, autopercepção e saúde e morbidades prevalentes em idosos. [Tese de doutorado. Pontifícia Universidade Católica do Rio Grande do Sul].

Rocha, L. M., Terra, N. (2013). Body image in older adults: a review. Scientia Medica, 23(4), pp. 255-261. https://doi.org/10.15448/1980-6108.2013.4.15357

Saez de Asteasu M. L., Matínez-Velilla, N., Zambom-Ferraresi, F., Casas-Herrero, A. \& Izquierdo, M. (2017). Role of physical exercise on cognitive function in healthy older adults: a systematic review of randomized clinical trials. Aging Res. Rev, 37, 117-134. https://www.ncbi.nlm.nih.gov/pubmed/28587957.

Scatolin, H. (2012). A imagem do corpo: as energias construtivas da psique. Psicologia Revista, 21(1), 115-120. https://revistas.pucsp.br/index.php/psicorevista/article/view/13586

Sofi, F., Valecchi, D., Bacci, D., Abbate, R., Gensini, G., Casini, A., \& Macchi, C. (2010). Physical activity and risk of cognitive decline: a meta-analysis of prospective studies. J Intern Med, 269, 107-117. https://www.ncbi.nlm.nih.gov/pubmed/20831630

Steglich, L. A. (1978). Terceira idade, aposentadoria, auto-imagem e auto-estima. [Dissertação de mestrado. Universidade Federal do Rio Grande do Sul]. 